DOI: http://dx.doi.org/10.22198/rys.2018.72.a839

Artículos

\title{
El bienestar social de la niñez mexicana según vínculos migratorios internacionales: una aproximación
}

\author{
Mexican children's social welfare \\ according to international migration links: \\ an approach
}

\author{
Mónica Chávez Elorza* \\ orcid.org/0000-0002-6687-9148 \\ Selene Gaspar Olvera \\ orcid.org/0000-0002-1312-5385 \\ Héctor Rodríguez Ramírez ${ }^{* * *}$ \\ orcid.org/0000-0002-0740-4265
}

Resumen: desde inicios del siglo XXI permanece una visión positiva de los efectos de la migración internacional en la promoción del desarrollo de los países de origen, especialmente

\footnotetext{
* Autora para correspondencia. Docente-investigadora. Unidad Académica en Estudios del Desarrollo, Universidad Autónoma de Zacatecas. Av. Preparatoria s/n, colonia Hidráulica, C. P. 98065, Zacatecas, Zacatecas, México. Correo electrónico: monica.chavez@estudiosdeldesarrollo. net

** Investigadora. Unidad Académica en Estudios del Desarrollo, Universidad Autónoma de Zacatecas. Av. Preparatoria s/n, colonia Hidráulica, C. P. 98065, Zacatecas, Zacatecas, México. Correo electrónico: selene_gaspar@yahoo.com.mx

*** Director de la maestría en administración pública y política pública. Escuela de Gobierno y Transformación Pública del Instituto Tecnológico y de Estudios Superiores de Monterrey. Edificio EGAP. Av. Eugenio Garza Lagüera esquina Av. Rufino Tamayo s/n, C. P. 66269, San Pedro Garza García, Nuevo León, México.Teléfono: (81) 86258381 y 60. Correo electrónico: hrr@itesm.mx
} 
de las remesas. El objetivo aquí es examinar los indicadores de bienestar de los niños que viven en hogares con vínculos migratorios internacionales. Con los datos del censo de población y vivienda de 2010 y con la estadística descriptiva se revisan las condiciones sociales que prevalecen en dicha población, cuando en el hogar está presente uno de los padres o ambos. Los resultados muestran que la niñez mexicana enfrenta limitaciones con respecto al trabajo infantil, el rezago educativo, la inasistencia escolar y la insuficiencia alimentaria, y que quienes viven en hogares con vínculos migratorios son más vulnerables. Lo que implica que el Estado debe diseñar políticas públicas que consideren las características de esta población para mejorar su condición social y de desarrollo.

Palabras clave: bienestar social; migraciones internacionales, vivienda, infancia; México.

Abstract: since the start of the 21 st century, a positive view of the effects of international migration on promotion of development of origin countries, especially regarding remittances, remains. The aim here is to examine the welfare indicators of children living in households with international migration links. Based on data from the 2010 Population and Housing Census and descriptive statistics, social conditions that prevail in this population are reviewed, with one of the parents, or both, being present in the household. The results show that Mexican children face limitations concerning child labor, educational backwardness, non-attendance at school and food insufficiency, and that those living in households with migration links are more vulnerable. This means that the State should design public policies that consider the characteristics of this population in order to improve its social condition and development.

Keywords: social welfare; international migrations; housing; childhood; Mexico. 
Recibido el 31 de agosto de 2016. Aceptado el 26 de abril de 2017.

\section{Introducción}

El estudio teórico y empírico del nexo entre migración y desarrollo ha oscilado, como un péndulo, de visiones positivas a negativas y viceversa (De Haas 2012). Una de las razones principales que subyace en esta oscilación es el monto de las remesas enviadas a los países en vías de desarrollo que, más allá de proporcionar estabilidad macroeconómica a los receptores, propicia el debate sobre si este dinero puede promover el bienestar social y económico individual y colectivo de las comunidades.

En este contexto surge la pregunta: ¿cómo se puede conocer la condición de bienestar social de la niñez según su conexión migratoria?, que es muy relevante en el caso mexicano, porque la emigración hacia Estados Unidos data de finales del siglo XIX, y en décadas recientes ha crecido, además de que otras poblaciones de zonas distintas a las tradicionales del occidente y centro-norte del país se han sumado a estos flujos migratorios (Delgado y Rodríguez 2004). Al mismo tiempo, desde la implementación de la política securitista de Estados Unidos y el cierre de su frontera, se ha incrementado el número de retornados (voluntarios o no) que, a su vez, ha propiciado nuevas configuraciones familiares dentro de los hogares vinculados a dicho fenómeno (Gaspar y Chávez 2016).

En este artículo se trata de responder a la pregunta planteada y para ello se analiza, de manera indirecta, si los menores con vínculos migratorios internacionales exhiben mejores indicadores de bienestar social: rezago educativo, trabajo infantil y juvenil y carencia de acceso a alimentos, respecto a los que no cuentan con ellos. Esto se examina descriptivamente, a partir de la ausencia que registran los niños, niñas y adolescentes de dichos indicadores, en tanto el papel de la migración y las remesas se vinculan con ellos a partir del hogar. La fuente de información primaria para esta investigación es la muestra del censo de población y vivienda de 2010, del Instituto Nacional de Estadística y Geografía (INEGI 2011). 
El texto se organiza en cuatro secciones; en la primera se revisa la literatura sobre el nexo entre migración, remesas y desarrollo -sobre indicadores precisos como asistencia escolar, trabajo infantil y pobreza-. Después se presentan los datos utilizados y el tratamiento metodológico de éstos. La tercera incluye el análisis de los indicadores de bienestar seleccionados y una discusión de sus implicaciones de política pública, y en la última se presentan las conclusiones.

\section{Migración internacional, remesas y desarrollo: revisión teórica}

En las últimas cinco décadas, el estudio del nexo entre migración y desarrollo se ha movido entre cuatro fases obedeciendo tanto a corrientes teóricas como a su visión positiva o negativa del efecto de la migración en el desarrollo de los países de origen (De Haas 2012; 2008; 2007, 4).

En la primera fase, hasta antes de 1973, la posición predominante establecía que la migración era un factor potencial para la promoción del desarrollo de los países emisores, a través de trasferencia de capital y conocimientos. Desde luego que esta visión asume un fuerte proceso de migración circular y de retorno.

En la segunda, entre 1973 y 1980, en los países emisores de emigrantes se empezó a registrar un desencanto con la migración como palanca del desarrollo, pues los migrantes y las remesas no lograban potenciar el desarrollo económico y social de sus lugares de origen. Asociado con este proceso se comenzó a hablar del brain waste o "fuga de cerebros", de pérdida de capacidades y de efectos económicos marginales.

En la tercera, en la década 1990-2000, se evidenció una dualidad en la visión de la migración y el desarrollo; algunos países, sobre todo de América Central y organismos de ayuda multilateral, como el Banco Interamericano de Desarrollo, replantearon la migración como palanca de desarrollo. Pero, por otra parte, se profundizó la visión de la migración como un factor de bajo impulso para el desarrollo en el país de origen. 
En la cuarta, durante la primera década del presente siglo, resurgió la visión optimista de la relación migración y desarrollo, debido al crecimiento exponencial de las remesas en varios países, el brain gain o la "circulación de talentos o cerebros", la trasferencia de capacidades y el capital financiero. Desde luego que aquí también se retoma la idea de una migración circular y de retorno.

Desde una perspectiva más crítica, Canales (2015a; 2015b) señala que el debate sobre migración y desarrollo subsume un análisis más importante sobre: a) las causas y factores que desencadenan la migración internacional; b) los impactos y la invisibilidad de las contribuciones de la migración en los países de destino; c) la distorsión de los derechos versus los números de los inmigrantes y d) los costos y las repercusiones de la emigración en los países de origen, como la sobrevaloración de las remesas; en este sentido, apunta que éstas no compensan los costos económicos y sociales de reproducción de los trabajadores emigrantes (2015a). Por lo que la discusión se debe dejar de centrar en la "falta de desarrollo", para hacerlo en el "estilo de desarrollo"; esto es, el estudio de la migración debe estar circunscrito en los procesos del modelo de producción capitalista contemporáneo, que la impulsa reforzando estructuras de desigualdad social en el mundo global (Canales 2015b).

Las implicaciones de estas perspectivas recaen en el rol que debe asumir el Estado, si es responsable o corresponsable del despegue del desarrollo de las comunidades y, por tanto, del tipo de políticas públicas que debe implementar. Para Castles y Delgado (2007), las remesas nunca podrán sustituir el rol de Estado y del mercado (del capital privado) en la promoción de la modernización productiva y de las trasformaciones estructurales, necesarias para impulsar el crecimiento económico.

\section{Migración internacional y el bienestar social: revisión empírica}

Diversos trabajos han mostrado la relación o el efecto entre la migración, las remesas y el bienestar social, este último visto como la disminución del trabajo infantil, la presencia de menores en las escuelas 
y la reducción de la pobreza. Los resultados de estas investigaciones han puesto en evidencia que no existe una relación unívoca y, por el contrario, que se trata de un fenómeno complejo determinado por contextos socioeconómicos y culturales multidimensionales.

Taylor y Wyatt (1996) muestran, en estudios realizados en diversos países, que las remesas recibidas de familiares que trabajan en el extranjero inducen a una mayor retención escolar y una disminución en el trabajo infantil. Por ejemplo, sugieren que en Pakistán, El Salvador, Nicaragua y otras economías latinoamericanas la migración tiende a reducir la incidencia de trabajo infantil, lo que favorece las posibilidades de que los niños asistan a la escuela.

Otras investigaciones evidencian que la migración y las remesas pueden ser factores disruptivos de la dinámica familiar, y generar efectos negativos sobre la asistencia escolar (Kandell y Kao 2001; McKenzie y Rapoport 2006; McKenzie y Sasin 2007). En Filipinas, México y El Salvador las remesas han tenido efectos negativos en la asistencia escolar y el trabajo infantil, pues cuando los hogares cuentan con mayor ingreso se les abren oportunidades de negocio, y esto incrementa el costo de oportunidad para que sus miembros asistan a la escuela, lo que propicia el aumento del trabajo infantil; se pueden encontrar ejemplos de esto en Acosta (2006); Hanson y Woodruff (2003) y Boucher et al. (2005).

En un sentido más amplio, Adams y Page (2005) analizaron el impacto de la migración y de las remesas en la disminución de la pobreza en 74 países en desarrollo. Sus resultados indican que la migración internacional, medida como la proporción de población de un país que vive en el exterior, reduce la pobreza en el de origen dado que, en promedio, un incremento de 10 por ciento de los emigrantes disminuirá en 1.9 la proporción de personas que viven con menos de un dólar al día. Asimismo, un aumento de 10 por ciento en las remesas reducirá en 2 por ciento la profundidad y la severidad de la pobreza en el mundo.

En México se ha analizado el impacto de las remesas en la asistencia escolar, escolaridad acumulada o logro académico de los individuos expuestos a la migración internacional México-Estados Unidos (Rapoport y Docquier 2005). 
Hanson y Woodruff (2003), con datos del Censo de población y vivienda de 2000, analizaron la escolaridad acumulada de las personas de 10 a 15 años en las áreas rurales. Sus resultados muestran que los niños y jóvenes que viven en hogares migrantes acumulan más escolaridad: los de 10 a 12 logran 0.8 años, en promedio, por cada año adicional de edad, y los de 13 a 15 acumulan sólo 0.6 años. Por otro lado, encontraron que si el hogar recibe o no remesas incide en la asistencia escolar de los niños en las familias cuyos padres tienen muy pocos estudios. López (2004) sostiene que las remesas que provienen de los migrantes internacionales están asociadas con mejoras en el desarrollo. Con datos municipales, encontró que un incremento en la fracción de los hogares que reciben remesas está correlacionado con mejoras en los indicadores de salud, de educación y con reducciones de la pobreza: disminuye la mortalidad infantil y el analfabetismo en los niños de 6 a 14 años de edad, y aumenta la asistencia escolar de los jóvenes de 14. Por último, McKenzie y Rapoport (2006) usan la Encuesta nacional de la dinámica demográfica 1997, para analizar a niños y jóvenes de 12 a 18 años que viven en 12980 hogares rurales; la migración redujo el logro educativo y la asistencia escolar en los jóvenes de 12 a 18 y en los de 16 a 18.

Es importante destacar que en estas investigaciones no se realiza un análisis a profundidad del efecto de la ausencia de uno o de ambos padres en los hogares estudiados. Dicho factor es relevante puesto que puede ser benéfico o perjudicial para la asistencia escolar, el logro educativo y la inserción en el mercado laboral de los niños y jóvenes. Por ejemplo, se puede suponer que si un padre está ausente los jóvenes pudieran seguir estudiando, si éste les proporciona los recursos monetarios para el sostenimiento del hogar, para que a su vez la estructura familiar no les demande que trabajen, para compensar la ausencia del padre. De lo contrario, cuando el padre no está y el dinero es insuficiente, el joven tendría que laborar, y abandonar la escuela. No obstante, es importante señalar que este factor es uno de los muchos que inciden en la inserción y permanencia escolar de los menores y los jóvenes con vínculos migratorios internacionales.

Canales (2006) sostiene una postura distinta, utiliza datos de la Encuesta nacional de ingreso y gasto de los hogares 2002 para mostrar que en México, aunque las remesas contribuyen a mejorar las 
condiciones de calidad de vida de los hogares receptores, no disminuyen sus condiciones de pobreza y desigualdad. Él sustenta su planeamiento en que estas remesas son salarios internacionales $y$, por tanto, no existe motivo alguno para que se gasten de un modo distinto al de cualquier otra fuente de ingreso; su función sigue siendo garantizar la reproducción de la mano de obra.

\section{Los datos y su tratamiento metodológico}

Aquí se utilizan los datos del Censo de población y vivienda 2010 (INEGI 2011) y no los de la Encuesta intercensal 2015, debido a que no incluyen la batería de preguntas del módulo de migración internacional. La definición del hogar con vínculos migratorios se basa en la propuesta metodológica de Gaspar (2017), quien parte de la definición de hogar censal del INEGI $(2011,125-128)$ y operacionaliza la definición de un hogar con vínculos migratorios internacionales como el que cuenta con un jefe(a) nacido(a) en México y que al menos uno de sus miembros tenga antecedentes migratorios hacia Estados Unidos u otras partes del mundo y que en éste haya al menos una persona que: a) en los cinco años previos al levantamiento censal viviera fuera de México; b) recibe remesas internacionales; c) es un emigrante internacional del quinquenio inmediato anterior al levantamiento censal, independientemente de que haya retornado o no, y d) sea nacida fuera del país, pero de origen mexicano. Una vez definido el tipo de hogar, se identifica a los menores que pertenecen a él (Gaspar 2017). Por tanto, la unidad de análisis son los menores y no los hogares.

La vinculación de los menores o de cualquier otro grupo con la emigración internacional, a través del hogar, permite conocer, hasta cierto punto, cómo opera este fenómeno demográfico en las condiciones sociales y económicas (Gaspar 2017), e identificar necesidades apremiantes, es decir, verlo como una aproximación a las condiciones de bienestar social de la niñez mexicana (personas de 17 años o menos), que se analizan considerando la carencia que se manifiesta en tres indicadores: rezago educativo, trabajo infantil y carencia de acceso a alimentos. 
Los datos del INEGI (2011) revelan que de los 28696180 hogares que había en México, 18068869 (63 por ciento) tenían menores de 18 años entre sus miembros, y en 1587811 (8.8) de éstos había vínculos migratorios internacionales. La población objetivo fue de 36251448 personas, de entre 12 a 17 años, y 3328317 (9.2 por ciento) de ellas vivían en hogares con vínculos migratorios internacionales; en 31.6 por ciento de éstos estaba presente uno de los padres, mientras que los menores que vivían en otro tipo de hogar representaban 17.3 por ciento. Asimismo, 7.1 por ciento de los menores con vínculos migratorios tenían a ambos padres ausentes, y este porcentaje era de 3.7 para quienes residían en otro tipo de hogar (véase Figura 1).

Figura 1. Menores de 18 años con y sin vínculos migratorios internacionales, 2010

\begin{tabular}{|c|r|r|r|r|r|}
\hline & \multirow{2}{*}{ Total } & \multicolumn{2}{|c|}{$\begin{array}{c}\text { Ambos } \\
\text { presentes }\end{array}$} & $\begin{array}{c}\text { Sólo uno } \\
\text { presente }\end{array}$ & $\begin{array}{c}\text { Ambos } \\
\text { ausentes }\end{array}$ \\
\cline { 3 - 6 } & & 36251448 & 28045041 & 6752770 & 1453637 \\
\hline Menores en hogares (personas) & 3328131 & 2042734 & 1050614 & 234783 \\
\hline Con vínculos migratorios & 32923317 & 26002307 & 5702156 & 1218854 \\
\hline Otro tipo de hogar & 100.0 & 77.4 & 18.6 & 4.0 \\
\hline Menores en hogares (porcentajes) & 100.0 & 61.4 & 31.6 & 7.1 \\
\hline Con vínculos migratorios & 100.0 & 79.0 & 17.3 & 3.7 \\
\hline Otro tipo de hogar & & &
\end{tabular}

Fuente: estimación propia, con base en el INEGI (2011).

En la Figura 2 se incluyen las características de niñas, niños y adolescentes que vivían en hogares con vínculos migratorios versus los que residían en los de otro tipo, y son muy semejantes en cuanto a la presencia del(los) padre(s); por ejemplo, en términos del sexo de los menores, prácticamente no hubo diferencias.

Con respecto a la distribución por grupos de edad y en donde sólo uno de los padres estaba presente, la diferencia fue ligera en los dos casos, que también se observó en el rubro de asistencia escolar. 
Figura 2. Características de los menores de 18 años con y sin vínculos migratorios internacionales según presencia de los padres, 2010

\begin{tabular}{|c|c|c|c|c|}
\hline \multirow[b]{2}{*}{ Menores } & \multirow{2}{*}{ Total } & \multicolumn{3}{|c|}{ Presencia de los padres } \\
\hline & & $\begin{array}{c}\text { Ambos } \\
\text { presentes }\end{array}$ & $\begin{array}{l}\text { Sólo uno } \\
\text { presente }\end{array}$ & $\begin{array}{c}\text { Ambos } \\
\text { ausentes }\end{array}$ \\
\hline \multicolumn{5}{|l|}{ Sexo } \\
\hline Con vínculos migratorios & 3328131 & 2042734 & 1050614 & 234783 \\
\hline Hombres & 51.1 & 51.1 & 51.3 & 50.7 \\
\hline Mujeres & 48.9 & 48.9 & 48.7 & 49.3 \\
\hline Sin vínculos migratorios & 32923317 & 26002307 & 5702156 & 1218854 \\
\hline Hombres & 51.0 & 51.0 & 51.1 & 51.6 \\
\hline Mujeres & 49.0 & 49.0 & 48.9 & 48.4 \\
\hline \multicolumn{5}{|l|}{ Grupos de edad } \\
\hline Con vínculos migratorios & 3328131 & 2042734 & 1050614 & 234783 \\
\hline De 0 a 11 años & 66.8 & 68.5 & 66.5 & 53.4 \\
\hline De 12 a 17 años & 33.2 & 31.5 & 33.5 & 46.6 \\
\hline Sin vínculos migratorios & 32923317 & 26002307 & 5702156 & 1218854 \\
\hline De 0 a 11 años & 67.2 & 68.8 & 62.9 & 52.4 \\
\hline De 12 a 17 años & 32.8 & 31.2 & 37.1 & 47.6 \\
\hline \multicolumn{5}{|c|}{ Asistencia escolar (6 años o más) } \\
\hline Con vínculos migratorios & 2223201 & 1315280 & 714522 & 193399 \\
\hline Asisten & 87.7 & 88.1 & 88.1 & 83.0 \\
\hline No asisten & 12.3 & 11.9 & 11.9 & 17.0 \\
\hline Sin vínculos & 22112414 & 17108408 & 4008792 & 995214 \\
\hline Asisten & 89.5 & 90.6 & 87.1 & 78.7 \\
\hline No asisten & 10.5 & 9.4 & 12.9 & 21.3 \\
\hline
\end{tabular}

Fuente: estimación propia, con base en el INEGI (2011). 


\section{Bienestar social de niñas, niños y adolescentes en México Inasistencia escolar y rezago educativo}

La Norma de Escolaridad Obligatoria del Estado Mexicano ${ }^{1}$ establece que la población con carencia por rezago educativo es la que cumpla alguno de los criterios siguientes: a) tener de 3 a 15 años, no contar con la educación básica obligatoria ni asistir a un centro de educación formal; b) haber nacido antes de 1982, y no haber cursado la educación obligatoria vigente en el momento en que debía haberlo hecho (primaria completa); c) haber nacido después de 1982, y no tener acreditada la educación obligatoria (secundaria completa), tener la edad y la escolaridad acumulada que debería de acuerdo con el sistema educativo mexicano.

En este trabajo se aproximó al rezago educativo mediante la observación de la asistencia escolar y el nivel de escolaridad que debería tener un menor por grupo de edad. La inasistencia sólo indica si la persona asiste o no a un centro educativo.

Los datos reflejan un porcentaje ligeramente mayor de inasistencia escolar en hogares con vínculos migratorios (12.3 por ciento), en comparación con los que no los tenían (10.5) (véase Figura 2). Esta tendencia se mantuvo al considerar las variantes de presencia de los padres en los hogares. Según los datos censales, hubo más rezago educativo en hogares con vínculos migratorios, con respecto a los que carecían de ellos (véase Figura 3).

Estos resultados muestran una relación compleja entre la inasistencia escolar, el rezago educativo, la migración y la presencia de los padres, de tal suerte que la ausencia de ambos padres tiene un efecto negativo en la asistencia escolar de los niños, por lo que en cualquiera de los rangos de edad analizados este factor potencia la mayor incidencia del fenómeno.

Aunque la diferencia en puntos porcentuales de la inasistencia y el rezago escolar en los dos tipos de hogares fue mínima, pudo dar cuenta de los factores estructurales que estaban operando, pero dicho análisis sobrepasa el alcance de este trabajo.

1 Consejo Nacional de Evaluación de la Política de Desarrollo Social. http://www.coneval.org. mx/Medicion/Paginas/Medici\%C3\%B3n/Avances-Rezago-educativo.aspx 
Figura 3. Menores entre 6 y 17 años con rezago educativo según vinculación migratoria (\%), 2010

\begin{tabular}{|c|c|c|}
\hline \multirow[b]{2}{*}{ Presencia de los padres y rezago } & \multicolumn{2}{|l|}{ Menores } \\
\hline & $\begin{array}{c}\text { Con vínculos migratorios } \\
\text { internacionales }\end{array}$ & No migrantes \\
\hline Total & 12.3 & 10.5 \\
\hline \multicolumn{3}{|c|}{ Presencia de los padres } \\
\hline Ambos presentes & 11.9 & 9.4 \\
\hline Sólo la madre & 11.4 & 12.3 \\
\hline Sólo el padre & 20.3 & 18.3 \\
\hline Ambos ausentes & 17.0 & 21.3 \\
\hline \multicolumn{3}{|c|}{ Rezago educativo } \\
\hline \multicolumn{3}{|c|}{6 a 11 años } \\
\hline Sin escolaridad & 23.2 & 22.8 \\
\hline Ambos padres presentes & 23.1 & 22.9 \\
\hline Sólo la madre & 23.7 & 22.6 \\
\hline Sólo el padre & 21.7 & 20.5 \\
\hline Ambos ausentes & 21.9 & 21.3 \\
\hline \multicolumn{3}{|c|}{12 a 17 años } \\
\hline Hasta primaria incompleta & 17.5 & 16.9 \\
\hline Ambos padres presentes & 17.1 & 16.6 \\
\hline Sólo la madre & 17.2 & $17.2^{* *}$ \\
\hline Sólo el padre & 18.8 & 18.0 \\
\hline Ambos ausentes & 21.0 & 19.6 \\
\hline
\end{tabular}

Nota: todos son estadísticamente diferentes.

** Indica que los pares de columnas de esa fila no son estadísticamente distintos con un nivel de significancia de 95 por ciento.

Fuente: estimación propia, con base en el INEGI (2011).

\section{Condición de actividad laboral}

La Constitución Políitica de los Estados Unidos Mexicanos señala en su artículo $3^{\circ}$ que "toda persona tiene derecho a la educación. El Estado -Federación, Estados, Ciudad de México y Municipios, impartirá educación básica, preescolar, primaria, secundaria, y que ésta y la educación media superior son obligatorias" (Cámara de Diputados 1917). Además, 
la Ley Federal del Trabajo (Cámara de Diputados 1970) establece en sus artículos 22, 22 Bis y 23 que "está prohibido el trabajo de los menores de 15 años, no podrán laborar los que tengan entre 15 y 18 años que no hayan terminado su educación básica obligatoria, salvo que la autoridad laboral señale compatibilidad entre trabajo y estudios". Pueden laborar los jóvenes de entre 15 y 16 años de edad con autorización de sus padres o tutores y a falta de ellos, con el permiso de la autoridad correspondiente. Está "prohibido el trabajo de los menores de 18 años dentro del círculo familiar en cualquier actividad peligrosa para su salud, seguridad o moralidad, que afecte sus derechos y con ello, su desarrollo integral".

Si se considera lo anterior, se espera que la proporción de jóvenes que labora sea mínima, no obstante es necesario precisar que los datos del INEGI (2011) no permiten saber si éstos laboran dentro del círculo familiar, aunque sí es posible estimar la proporción de los que trabajan y estudian, y la de quienes sólo trabajan, así como de aquéllos que no trabajan ni estudian.

La condición laboral de los jóvenes de 12 a 17 años pone de manifiesto la necesidad de sus familias de contar con un ingreso adicional; fue mayor la proporción de los que trabajaban y pertenecían a hogares con vínculos migratorios, que la de quienes vivían en hogares de no migrantes. Sin embargo, hubo contraste ante la presencia o la ausencia de ambos padres (véase Figura 4).

Figura 4. Menores entre 12 y 17 años según condición de actividad laboral y vinculación migratoria internacional (\%), 2010

\begin{tabular}{|c|c|c|c|c|}
\hline \multirow{3}{*}{$\begin{array}{c}\text { Presencia de } \\
\text { los padres }\end{array}$} & \multicolumn{2}{|c|}{ Trabaja } & \multicolumn{2}{|c|}{ Busca trabajo } \\
\hline & \multicolumn{2}{|c|}{ Menores } & \multicolumn{2}{|c|}{ Menores } \\
\hline & $\begin{array}{c}\text { Con vínculos } \\
\text { migratorios } \\
\text { internacionales }\end{array}$ & $\begin{array}{c}\text { No } \\
\text { migrantes }\end{array}$ & $\begin{array}{c}\text { Con vínculos } \\
\text { migratorios } \\
\text { internacionales }\end{array}$ & $\begin{array}{c}\text { No } \\
\text { migrantes }\end{array}$ \\
\hline Total & 14.0 & 11.0 & 1.4 & 1.3 \\
\hline Ambos presentes & 14.1 & 9.9 & 1.3 & 1.2 \\
\hline Sólo la madre & 13.3 & 12.5 & 1.4 & 1.6 \\
\hline Sólo el padre & 17.5 & 15.0 & 1.9 & 2.1 \\
\hline Ambos ausentes & 15.2 & 19.1 & 1.9 & 2.3 \\
\hline
\end{tabular}

Nota: todos son estadísticamente diferentes a nivel de significancia de 95 por ciento. Fuente: estimación propia, con base en el INEGI (2011). 


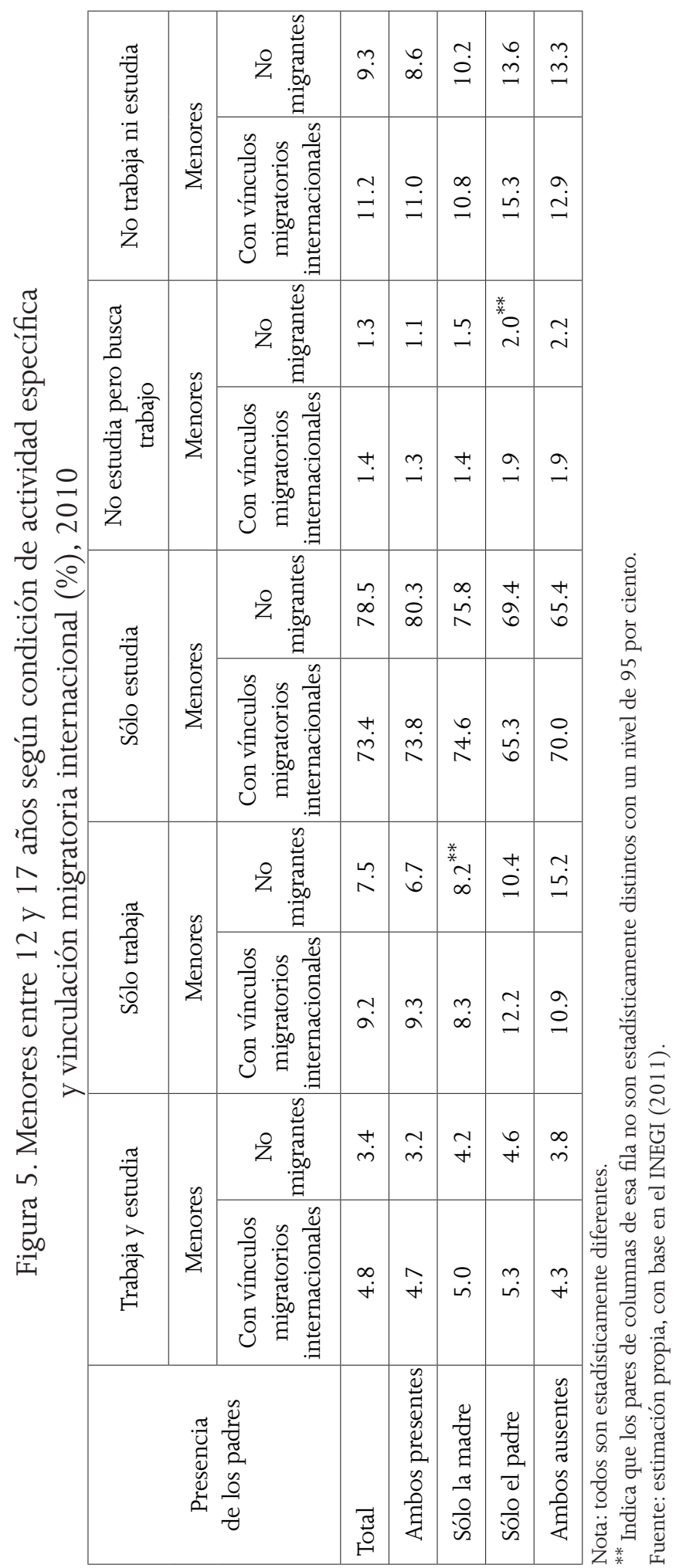


Los resultados evidencian que la condición de "no trabaja ni estudia” pone en discusión si es por falta de dinero o de recursos que los jóvenes no tienen alguna opción que les permita un desarrollo más integral, a través del estudio o de la experiencia laboral (véase Figura 5).

$\mathrm{Al}$ igual que en el ámbito de la educación y el rezago educativo, los datos referidos en las Figuras 4 y 5 ponen de manifiesto que el efecto positivo de los vínculos migratorios internacionales en la disminución del trabajo infantil es escaso. Si bien esta actividad está regulada por la Ley Federal del Trabajo, la realidad muestra el incumplimiento de este derecho social. Los datos reflejan porcentajes elevados de trabajo infantil, en particular en los menores vinculados con la migración internacional.

\section{Insatisfacción alimentaria}

De acuerdo con el artículo $6^{\circ}$ de la Ley General de Desarrollo Social, "el acceso a la alimentación nutritiva de calidad, junto con la educación, la salud, la vivienda, el disfrute de un medio ambiente sano, el trabajo y la seguridad social y los relativos a la no discriminación", forman parte de los derechos para el desarrollo social (Cámara de Diputados 2004). La encuesta asociada con el censo de 2010 del INEGI (2011) incluye preguntas como: ¿alguna persona de la vivienda se quedó sin comida, en los últimos tres meses, por falta de dinero o recursos?, ¿alguna de las personas de la vivienda comió una vez al día o dejó de hacerlo todo un día? (véase Figura 6).

El mismo censo reveló que casi uno de cada diez niños mexicanos pertenecía a hogares que habían padecido carencia de alimentos: 9.5 por ciento de los no migrantes registraron esta situación, en tanto que 8.5 de los menores con vínculos migratorios vivían en hogares con esa carencia. En los datos analizados hasta ahora, este es el único aspecto del bienestar social en el que para los niños con vínculos migratorios hubo algún efecto positivo diferenciador respecto del resto. Lo anterior permite sostener la hipótesis de que las remesas (internas e internacionales) y los apoyos gubernamentales tienen una incidencia mayor en la provisión de bienes de consumo (alimentos), al menos en los menores vinculados al fenómeno migratorio, que en los hogares que no lo están, y que sólo cuentan con remesas internas y apoyos gubernamentales (véase Figura 6 y 7 ). 


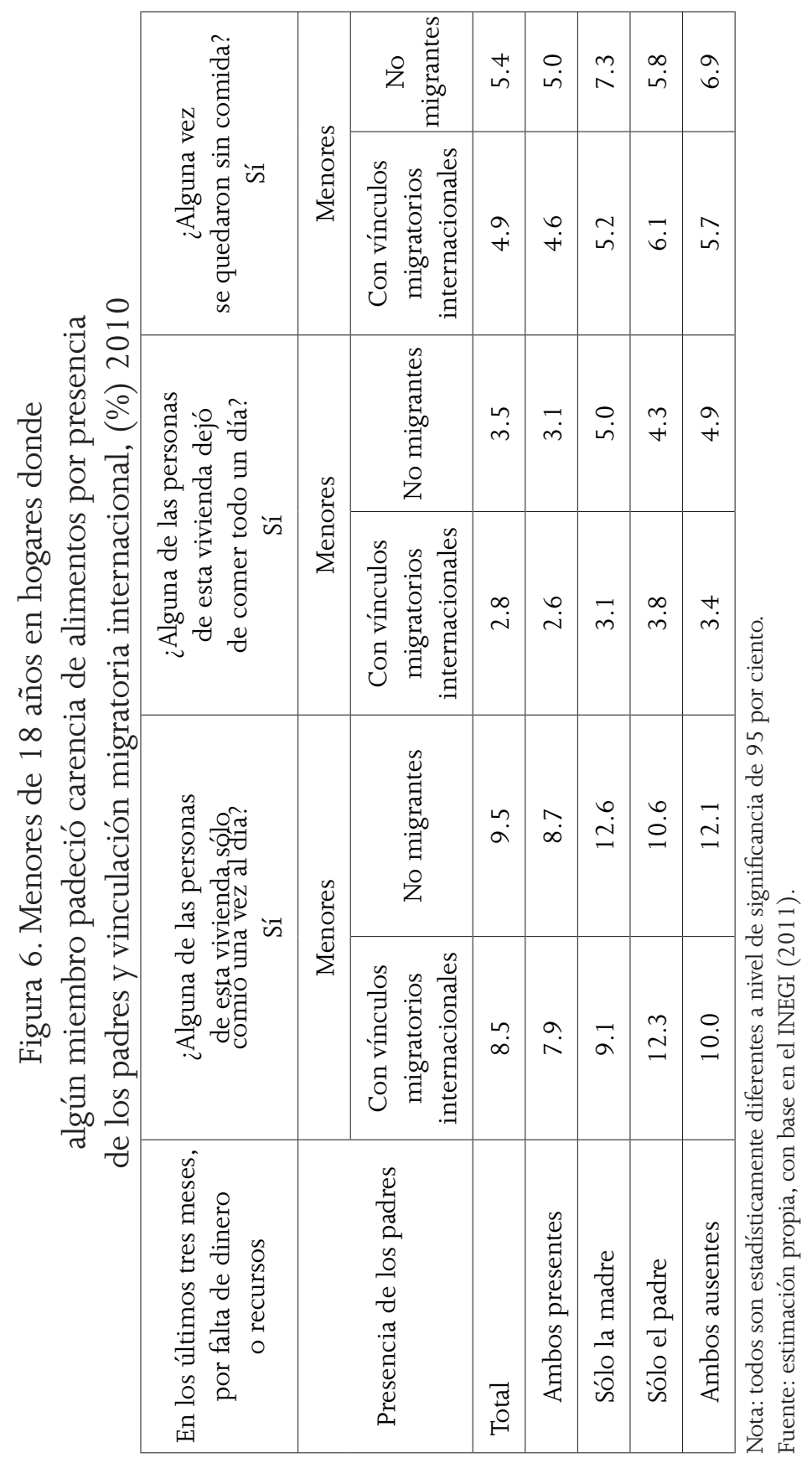




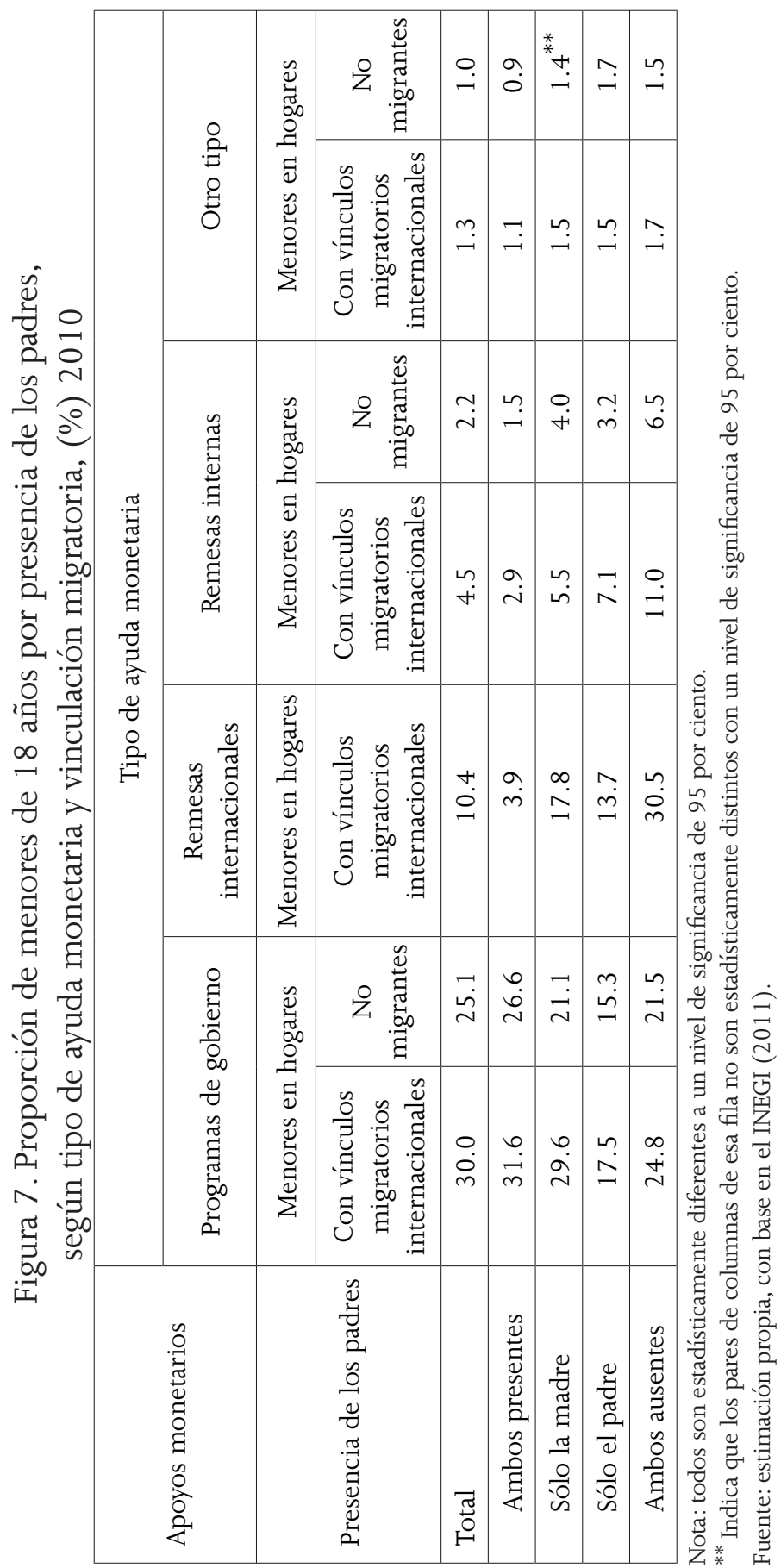




\section{Conclusiones}

Los datos empíricos utilizados en este trabajo, y que corresponden al INEGI (2011), sugieren que los menores con vínculos migratorios (3 328 131) no contaban con mejores niveles de bienestar que quienes vivían en hogares sin ellos (32 923 317).

Entre hogares con y sin vínculos migratorios, la inasistencia escolar y el rezago educativo de la niñez seguían siendo altos y poco diferenciados entre ambos tipos de menores; 12.3 por ciento de los vinculados con la migración presentaron inasistencia escolar, y 10.5 quienes no lo estaban. En cuanto al rezago educativo, 23.2 por ciento de los niños de entre 6 y 11 años de los hogares con vínculos migratorios lo presentaban, en comparación con 22.8 de los que residían en hogares sin estos vínculos.

La intensidad del trabajo infantil fue más alta en los menores con vínculos migratorios, 14 por ciento versus 11 . Aunque es necesario señalar que la ausencia o la presencia de ambos padres mostró situaciones contrastantes entre los de ambos tipos de hogares.

Sigue presente la carencia de alimentos o situación de hambre que vive la niñez en muchos hogares mexicanos, aunque fue menor en los niños con vínculos migratorios (8.5 por ciento), que en los que no los tenían (9.5).

Por un lado, estos resultados reflejan que los derechos, aunque estén plasmados en la Constitución Política de los Estados Unidos Mexicanos, no son asequibles para todos de igual manera $y$, por otro, que la postura crítica de ver a las remesas como parte de un salario internacional garantiza la reproducción de mano de obra y, por tanto, su capacidad promotora del desarrollo es limitada. Esto pone de manifiesto la necesidad de que el Estado mexicano implemente un modelo de desarrollo que potencie sus programas y acciones públicas para mejorar, más y con mayor eficiencia, el bienestar social y la calidad de vida de los mexicanos, especialmente de niñas, niños y adolescentes. 


\section{Bibliografía}

Acosta, Pablo. 2006. Labor supply, school attendance, and remittances from international migration: the case of El Salvador. World Bank Policy Research Working paper 3903.

Adams, Richard y John Page. 2005. El impacto de la migración internacional y de las remesas en la pobreza. En Las remesas, su impacto en el desarrollo y perspectivas futuras, editado por Samuel M. Munzele y Ratha Dilip, 261-286. Colombia: Banco Mundial y Mayol Ediciones.

Bouhcer, Steve, Oded Stark y J. Edward Taylor. 2005. A gain with a drain? Evidence from rural Mexico on the new economics of the brain drain, ZEF Discussion Papers on Development Policy. No. 99. http://www.zef.de/uploads/tx_zefportal/Publications/zef_ dp99.pdf (12 de febrero de 2018).

Cámara de Diputados. 1970. Ley Federal del Trabajo. Última reforma publicada en el Diario Oficial de la Federación. 12-06-2015. http:// www.dof.gob.mx/nota_detalle.php?codigo $=5396526 \&$ fec ha=12/06/2015 (10 de enero de 2017).

Cámara de Diputados. 2004. Ley General de Desarrollo Social. Última reforma publicada en el Diario Oficial de la Federación. 01-06-2016. http://www.dof.gob.mx/nota_detalle.php?codigo=5439561\&fec ha $=01 / 06 / 2016$ (10 de enero de 2017).

Cámara de Diputados. 1917. Constitución Política de los Estados Unidos Mexicanos. Última reforma publicada en el Diario Oficial de la Federación. 1508-2016. http://www.dof.gob.mx/nota_detalle.php?codigo $=54$ 47903\&fecha $=15 / 08 / 2016$ (10 de enero de 2017).

Canales, Alejandro. 2015a. El debate sobre migración y desarrollo. Evidencias y aportes desde América Latina. Latin American Research Review 50 (1): 29-52. 
Canales, Alejandro. 2015b. E PUR SI MUOVE. Elementos para una teoría de las migraciones en el capitalismo global. México: Universidad de Guadalajara y Miguel Ángel Porrúa.

Canales, Alejandro. 2006. El papel de las remesas en la reducción de la pobreza en México. Mitos y realidades. Carta Económica Regional 19 (98): 3-12.

Castles, Stephen y Raúl Delgado Wise. 2007. Introducción. Migración y desarrollo: perspectivas desde el sur. En Migración y desarrollo: perspectivas desde el sur, coordinado por Stephen Castles y Raúl Delgado Wise, 5-19. México: Miguel Ángel Porrúa.

De Haas, Hein. 2012. The migration and development pendulum: a critical review on research and policy. International Migration 50 (3): 8-25.

De Haas, Hein. 2008. Migration and development. A theoretical perspective. International Migration Institute. James Martin 21 st Century School University of Oxford Working paper 9. https://www. imi.ox.ac.uk/pdfs/wp/wp-09-08.pdf

De Haas, Hein. 2007. Remittances, migration and social development. A conceptual review of the literature. Social Policy and Development Programme paper 34 UNRISD. http://www.unrisd. org / 80256B3C005BCCF9/(httpAuxPages)/8B7D005E37FFC77 EC12573A600439846/\$file/deHaaspaper.pdf

Delgado Wise, Raúl y Héctor Ramírez. 2002. El nuevo panorama de la migración internacional y sus potencialidades para el desarrollo regional en Zacatecas. En El norte de todos: migración y empleo en tiempos de globalización, compilado por Jesús Arroyo, Alejandro Canales y Patricia Vargas, 209-238. México: Universidad de Guadalajara, Universidad de California Los Ángeles y Juan Pablos editor.

Gaspar-Olvera, Selene. 2017. Vulnerabilidad de los hogares con vínculos migratorios internacionales en México. Revista Diarios de Terruño 3: $13-38$. 
Gaspar-Olvera, Selene y Mónica Chávez-Elorza. 2016. Efecto de la migración internacional México-Estados Unidos: hogares con vínculos migratorios y su caracterización en cada país. ODISEA. Revista de Estudios Migratorios 3: 124-150.

Hanson, Gordon H. y Christopher Woodruff. 2003. Emigration and educational attainment in Mexico. University of California, San Diego, mimeo. http://www.childmigration.net/files/Hanson_2003.pdf

INEGI. 2015. Encuesta intercensal 2015. http://www.beta.inegi.org. $\mathrm{mx} /$ proyectos/enchogares/especiales/intercensal/

INEGI. 2011. Censo de población y vivienda 2010: marco conceptual. México.

INEGI. 2002. Encuesta nacional de ingresos y gastos de los hogares 2002. http://www.beta.inegi.org.mx/proyectos/enchogares/regulares/enigh/tradicional/2002/default.html

INEGI. 1997. Encuesta nacional de la dinámica demográfica 1997. http://www.beta.inegi.org.mx/proyectos/enchogares/especiales/enadid/1997/

Kandell, William y Grace Kao. 2001. The impact of temporary labor migration on Mexican children's educational aspirations and performance. International Migration Review 35 (4): 1205-1231.

López C., Ernesto. 2004. Globalization, migration and development: the role of Mexican migrant remittances. Inrter-American Development Bank. http://www.rrojasdatabank.info/iadbremit/globmigdev.pdf

McKenzie, David y Marcin J. Sasin. 2007. Migration, remittances, poverty, and human capital: conceptual and empirical challenges. Policy Research Working paper series 4272, The World Bank. 
McKenzie, David y Hillel Rapoport. 2006. Can migration reduce educational attainment? Evidence from Mexico. World Bank Policy Working paper No. 3952. The World Bank, Washington. http:// siteresources.worldbank.org/DEC/Resources/Can_Migration_reduce_Educational_Attainment.pdf

Rapoport, Hillel y Frédéric Docquier. 2005. The economics of migrants' remittances. IZA DP No. 1531. http://ftp.iza.org/dp1531. pdf

Taylor, J. Edward y Tom J. Wyatt. 1996. The shadow value of migrant remittances, income and inequality in a household-farm economy. The Journal of Development Studies 32 (6): 899-912.

World Bank. 2010. Can migration reduce educational attainment? Evidence from Mexico. Journal of Population Economics. Online First ${ }^{\mathrm{TM}}$. 